Pacific Journal of Mathematics

CONTINUOUS IMAGES OF WEAKLY COMPACT SUBSETS OF Yon BenYamin, Mary ElLEN Rubin and Michael L. WaGe 


\title{
CONTINUOUS IMAGES OF WEAKLY COMPACT SUBSETS OF BANACH SPACES
}

\author{
Y. Benyamini, M. E. Rudin And M. Wage
}

We give a positive answer to a problem of Lindenstrauss by showing that the family of compact Hausdorff spaces which are homeomorphic to weakly compact subsets of Banach spaces (Eberlein compacts) is stable under continuous images. This is equivalent to the fact that a Banach space $E$ is a subspace of a $W C G$ space iff the unit ball of $E^{*}$ is an Eberlein compact when equipped with the $w^{*}$-topology. We also study some topological properties of Eberlein compacts.

Introduction. A compact Hausdorff space is called an Eberlein compact $(E-C)$, if it is homeomorphic to a weakly compact subset of a Banach space. The survey paper [7] gives a detailed account of $E-C$ and the Banach spaces that they generate.

The main result of this paper is the following:

Theorem 2.1. A continuous image of an $E-C$, is also an $E-C$.

This gives a positive answer to problem 4 in [7]. In Banach space terms, the theorem is equivalent to the following characterization of subspaces of $W C G$ Banach spaces: $E$ is a subspace of a $W C G$ space iff $B\left(E^{*}\right)$-the unit ball of $E^{*}$ with its $w^{*}$-topology, is an $E-C$.

Essential to our work is the following purely topological characterization of $E-C$, due to H. P. Rosenthal [8]:

A compact Hausdorff space is an $E-C$ iff it has a $\sigma$-point-finite separating family of open $F_{\sigma}$ subsets.

Although this is a fairly easy reformulation of a theorem of Amir and Lindenstrauss (see $\S 1$, where we also define all the terms), this characterization represents an essential change of scene. Previous results were almost exclusively in the linear-topological context, while the problem at hand is topological and requires topological terminology and approach.

After some preliminaries in $\S 1$, we prove the main result in $\S 2$. In $\S 3$ we treat weakly compact subsets of Hilbert space. We show that this family is also stable under continuous images. We also give a characterization of such spaces in terms of the geometry of their space of continuous functions.

In $\S 4$ we prove some "nice" topological properties of $E-C$. We 
show that their cardinal functions are easily computed, and that an $E-C$ has "many" $G_{\delta}$ points.

Examples are given in $\$ 5$ and some problems are listed in the last section.

1. Preliminaries. If $\Gamma$ is a set, we denote by $c_{0}(\Gamma)$ the Banach space of all functions $f$ on $\Gamma$, such that for all $\epsilon>0$, the set $\{\gamma \in \Gamma:|f(\gamma)| \geqq \epsilon\}$ is finite. The norm on $c_{0}(\Gamma)$ is the sup norm. The weak topology on $c_{0}(\Gamma)$, when restricted to a weakly compact subset is the topology of pointwise convergence on $\Gamma$.

The main structure theorem for $E-C$ is due to D. Amir and J. Lindenstrauss [1]:

A compact Hausdorff space is an $E-C$, iff it is homeomorphic to a weakly compact subset of $c_{0}(\Gamma)$ for some set $\Gamma$.

In other words, every $E-C$ is homeomorphic to a compact subset $X$ of a large cube $[0,1]^{\mathrm{r}}$ with the product topology, where $X$ has the property that for each $\epsilon>0$ and $f \in X$ the set $\{\gamma \in \Gamma: f(\gamma) \geqq \epsilon\}$ is finite.

It is obvious, either from the definition or the above theorem that a closed subset of an $E-C$ is an $E-C$, and that a countable product of $E-C$ 's is an $E-C$. As remarked in [7] an uncountable product of non-trivial $E-C$ 's is never an $E-C$.

We have already mentioned Rosenthal's characterization of $E-C$ in the introduction. Let us just recall that a family $\mathscr{F}$ of subsets of $X$ is called separating, if given any $x \neq y$ in $X$, there is an $F \in \mathscr{F}$ such that either $x \in F$ and $y \notin F$ or $y \in F$ and $x \notin F$. The family $\mathscr{F}$ is called point-finite if each $x \in X$ belongs to at most finitely many sets in $\mathscr{F}$. It is called $\sigma$-point-finite if $\mathscr{F}=\bigcup_{n=1}^{\infty} \mathscr{F}_{n}$ where each $\mathscr{F}_{n}$ is point finite.

Definition. An $E-C, X$, is called strong if it embeds in $c_{0}(\Gamma)$ in such a way that $x(\gamma)=0$ or $x(\gamma)=1$ for all $x \in X$ and $\gamma \in \Gamma$. Equivalently, $X$ is a strong $E-C$ iff it has a point finite separating family of clopen sets.

Strong $E-C$ are much easier to handle and thus the following simple lemma (which was independently proved in [11]) will be very useful. We denote by $\mathbb{N}$ the natural numbers.

LEMMA 1.1. Every $E-C$ is a continuous image of a closed subset of $\prod_{n=1}^{\infty} X_{n}$, where each $X_{n}$ is a strong $E-C$.

Proof. Let $X$ be an $E-C$, and assume that $X$ is embedded in $c_{0}(\Gamma)$ in such a way that $0 \leqq x(\gamma) \leqq 1$ for all $x \in X$ and $\gamma \in \Gamma$. Thus $X$ is a closed subset of $[0,1]^{\Gamma}$ with the product topology. Let $\Lambda=\Gamma \times \mathbb{N}$ and define $\phi:\{0,1\}^{\Lambda} \rightarrow[0,1]^{\Gamma}$ by $(\phi(y))(\gamma)=\sum_{n=1}^{\infty} 2^{-n} y(\gamma, n)$. $\phi$ is a continuous onto map and $Y=\phi^{-1}(X)$ is a compact subset of $\{0,1\}^{\Lambda}$. For every 
$n$, let $R_{n}: Y \rightarrow\{0,1\}^{\wedge}$ be the restriction to $\Gamma \times\{n\}$, and define $X_{n}=$ $R_{n} Y$. Certainly $Y \subset \prod_{n=1}^{\infty} X_{n}$, and each $X_{n}$ is a strong $E-C$. Indeed, if $y \in Y$, there are at most finitely many $\gamma$ 's such that $(\phi(y))(\gamma) \geqq 1 / 2^{n}$, and if $\beta$ is not one of these, then necessarily $y(\beta, n)=0$.

We shall denote by $|A|$ the cardinality of the set $A$.

Definition. An $E-C, X$, is called uniform if it embeds in $c_{0}(\Gamma)$ in such a way that there is a function $N(\epsilon)$ such that for all $x \in X$ and $\epsilon>0$, $|\{\gamma:|x(\gamma)| \geqq \epsilon\}| \leqq N(\epsilon)$. Equivalently, $X$ is a uniform $E-C$ iff it has a separating family $\mathscr{F}$ of open $F_{\sigma}$ subsets for which there is a function $N(n)$ and a decomposition $\mathscr{F}_{F}=\cup \mathscr{F}_{n}$, such that each $x \in X$ belongs to at most $N(n)$ sets in $\mathscr{F}_{n}$.

Uniform $E-C$ were introduced in [2], where it was shown that $X$ is a uniform $E-C$ iff it is homeomorphic to a weakly compact subset of a Hilbert space. Also, an example was constructed in [2] of an $E-C$ which is not uniform.

Let $\Gamma$ be a set and denote by $\mathscr{K}(n)$ the subset of $\{0,1\}^{\Gamma}$ of all $x$ such that $|\{\gamma: x(\gamma)=1\}| \leqq n . \quad \mathscr{K}(n)$ is a strong and uniform $E-C$, and $\mathscr{K}=\mathscr{K}(1)$ is homeomorphic to the one point compactification of $\Gamma$.

For each $n, \mathscr{K}(n)$ is a continuous image of $\mathscr{K}^{n}$. Indeed, if $x=$ $\left(x_{1}, \ldots, x_{n}\right) \in \mathscr{K}^{n}$, then for each $i$ there is at most one $\gamma_{i}$ with $x_{i}\left(\gamma_{t}\right)=$ 1. Define $\varphi: \mathscr{K}^{n} \rightarrow \mathscr{K}(n)$ by

$$
(\varphi(x))(\gamma)= \begin{cases}1 & \gamma=\gamma_{i} \text { for some } 1 \leqq i \leqq n \\ 0 & \text { otherwise }\end{cases}
$$

It is easy to check that $\varphi$ is an onto continuous map. We shall denote by $\mathscr{K}^{\mathrm{N}}$ the product of countably many copies of $\mathscr{K}$. In the case of uniform $E-C$ we can now strengthen Lemma 1.1:

LEMMA 1.2. Every uniform $E-C$ is a continuous image of a closed subset of $\mathscr{K}^{\mathrm{N}}$.

Proof. We analyze the proof of Lemma 1.1. If $X$ is a uniform $E-C$ with function $N(\epsilon)$, we get that for each $y \in Y$ and $n$, there are at most $N\left(1 / 2^{n}\right) \gamma$ 's for which $\phi(y)(\gamma) \geqq 1 / 2^{n}$. Thus $R_{n} Y$ is not only a strong $E-C$, but in fact a subset of $\mathscr{K}\left(N\left(1 / 2^{n}\right)\right)$, and $Y$ is a closed subset of $\prod_{n=1}^{\infty} \mathscr{K}(n)$. But by the above remark each $\mathscr{K}(n)$ is a continuous image of $\mathscr{K}^{n}$ and thus $Y$ is a continuous image of a closed subset of $\Pi_{1}^{\infty} \mathscr{K}^{n}=\mathscr{K}^{\mathrm{N}}$.

REMARK. In Theorem 3.1 we show that the continuous image of a uniform $E-C$ is also a uniform $E-C$, and thus the above lemma 
characterizes uniform $E-C$, i.e., $X$ is a uniform $E-C$ iff it is a continuous image of a closed subset of $\mathscr{K}^{\mathrm{N}}$.

We finish this section by quoting a well known combinatorial lemma (see [5] or [6] p. 86-87 where a $\Delta$-system is called a quasi-disjoint family).

Definition. A family $\mathscr{A}=\left\{A_{\alpha}\right\}$ of subsets of $\Gamma$ is called a $\Delta$-system if there is a set $D \subset \Gamma$ such that $A_{\alpha} \cap A_{\beta}=D$ for all $\alpha \neq \beta$. The following lemma ensures that a given family $\mathscr{A}$ contains a "large" $\Delta$-system. Part (a) will be needed in $\$ 2$ and part (b) in $\$ 3$.

LEMMA 1.3. Let $\mathscr{A}$ be a family of subsets of a set $\Gamma$ such that $|A| \leqq n$ for some $n$ and all $A \in \mathscr{A}$.

(a) If $\mathscr{A}$ is infinite then $\mathscr{A}$ contains an infinite $\Delta$-system.

(b) There is a function $g(n, l)$ such that if $|\mathscr{A}| \geqq g(n, l)$, then $\mathscr{A}$ contains a $\Delta$-system of cardinality $l$.

2. Continuous images of $E-C$. In this section we prove the main result of this paper, namely

THEOREM 2.1. Let $X$ be an $E-C$ and $f$ a continuous function from $X$ onto $Y$. Then $Y$ is also an $E-C$.

Before giving the proof, we start with some lemmas and notation.

LEMMA 2.1. Let $Y$ be a compact Hausdorff space, and $\lambda$ the first ordinal with the same cardinality as $Y$. Let $U$ be an open subset of $Y$, and $S$ a relatively closed subset of $U$. Then there are open subsets $\{V(\alpha, m): \alpha<\lambda, m=1,2, \ldots\}$ of $U$, and compact subsets $S(\alpha, m)$ of $S \cap V(\alpha, m)$ such that

(a) $S=\cup\{S(\alpha, m): \alpha<\lambda, m=1,2, \ldots\}$

(b) If $\alpha>\beta$, then $S(\alpha, m) \cap V(\beta, n)=\varnothing$.

Proof. Index $Y$ as $Y=\left\{y_{\alpha}: \alpha<\lambda\right\}$. For each $\alpha<\lambda$ define inductively a sequence of compact subsets $K(\alpha, m)$ of $U$ and open subsets $V(\alpha, m)$ of $U$ as follows:

$$
K(\alpha, 1)=\left\{y_{\alpha}\right\} \quad \text { if } \quad y_{\alpha} \in U, \quad K(\alpha, 1)=\varnothing \quad \text { if } \quad y_{\alpha} \notin U .
$$

If $K(\alpha, m)$ has already been defined, let $V(\alpha, m)$ be an open set such that $K(\alpha, m) \subset V(\alpha, m) \subset \overline{V(\alpha, m)} \subset U$, and take $K(\alpha, m+1)=\overline{V(\alpha, m)}$.

Notice that for each $\alpha, \bigcup_{m=1}^{\infty} K(\alpha, m)=\bigcup_{m=1}^{\infty} V(\alpha, m)$ and hence is an open set. Thus $H(\alpha, m)=K(\alpha, m) \backslash \cup\{K(\beta, n): \beta<\alpha, n=$ $1,2, \ldots\}$ is compact. Define now $S(\alpha, m)=H(\alpha, m) \cap S$. Since $S$ is 
relatively closed and $H(\alpha, m)$ is compact, $S(\alpha, m)$ is compact. It is easy to check that these $S(\alpha, m), V(\alpha, m)$ satisfy (a) and (b).

Let now $X$ be an $E-C$ and $f: X \rightarrow Y$ an onto map. By Lemma 1.1 , we can assume that $X$ is a closed subset of a countable product of strong $E-C$, i.e. $X \subset\{0,1\}^{\Gamma}$ where $\Gamma=\bigcup_{n=1}^{\infty} \Gamma_{n}$, the $\Gamma_{n}$ are pairwise disjoint, and $\left\{\gamma \in \Gamma_{n}: x(\gamma)=1\right\}$ is finite for all $x \in X$ and all $n$.

For $x \in X$ we shall denote $\{\gamma \in \Gamma: x(\gamma)=1\}$ by $\Gamma(x)$, and $\Gamma(x) \cap\left(\bigcup_{n=1}^{k} \Gamma_{n}\right)$ by $\Gamma(x, k)$. The family of all finite subsets of $\Gamma$ will be denoted by $\mathscr{A}$.

For a fixed $A$ in $\mathscr{A}$, let

$$
\begin{aligned}
U(A) & =\left\{y \in Y: \Gamma(x) \cap A \neq \varnothing \text { for all } x \in f^{-1}(y)\right\} \\
S(A) & =\left\{y \in U(A): \forall \gamma \in A, \exists x \in f^{-1}(y) \text { with } \Gamma(x) \cap A=\{\gamma\}\right\} \\
& =U(A) \backslash \bigcup_{B \mp A} U(B) .
\end{aligned}
$$

Each $U(A)$ is an open subset of $Y$ and $S(A)$ is a relatively closed subset of $U(A)$.

Lemma 2.2. The family $\{U(A): A \in \mathscr{A}\}$ separates the points of $Y$. Moreover, if $w, y \in Y$ with $w \neq y$, then there is an $A \in \mathscr{A}$ such that either $y \in S(A)$ and $w \notin U(A)$, or $w \in S(A)$ and $y \notin U(A)$.

Proof. Fix $w \neq y$ in. $Y$. Then there is a $z \in f^{-1}(w)$ such that $\Gamma(x) \cap(\Gamma \backslash \Gamma(z)) \neq \varnothing$ for all $x \in f^{-1}(y)$ (or visa versa). For otherwise we could find $z_{n} \in f^{-1}(w)$ and $x_{n} \in f^{-1}(y)$ with $\Gamma\left(z_{n}\right) \supset \Gamma\left(x_{n}\right) \supset \Gamma\left(z_{n+1}\right) \supset$ $\cdots$. But then $x_{n}$ and $z_{n}$ have a common limit which is impossible.

Fixing $z$ as above, there is a finite subset $B$ of $\Gamma$, disjoint from $\Gamma(z)$, such that $\Gamma(x) \cap B \neq \varnothing$ for all $x \in f^{-1}(y)$. Indeed, if this were not the case, we could find inductively a sequence $x_{n} \in f^{-1}(y)$ such that for all $k<n, \Gamma\left(x_{k}, n\right) \cap \Gamma\left(x_{n}\right) \subset \Gamma(z)$. But then if $x$ is a limit point of $\left\{x_{n}\right\}$, we would have $\Gamma(x) \subset \Gamma(z)$, contradicting the choice of $z$.

Thus there is a minimal subset $A \subset B$ with the property that $\Gamma(x) \cap A \neq \varnothing$ for all $x \in f^{-1}(y)$. This is the desired $A$. Indeed, the condition $\Gamma(x) \cap A \neq \varnothing$ for all $x \in f^{-1}(y)$, means that $y \in U(A)$, and the minimality of $A$, implies that in fact $y \in S(A)$. However $w \notin U(A)$, since $z \in f^{-1}(w)$ and $\Gamma(z) \cap A=\varnothing$.

Lemma 2.3. Let $V \subset Y$ be open, and $S \subset V$ compact. Then there is a $k$ such that if $z \in X$ is such that there is a $t \in f^{-1}(S)$ with $\Gamma(z, k)=$ $\Gamma(t, k)$, then $z \in f^{-1}(V)$. 
Proof. If not, find for each $k, z_{k} \notin f^{-1}(V)$ and $t_{k} \in f^{-1}(S)$ with $\Gamma\left(z_{k}, k\right)=\Gamma\left(t_{k}, k\right)$. By compactness we can assume that $z_{k} \rightarrow z$, and then clearly also $t_{k} \rightarrow z$. However $X \backslash f^{-1}(V)$ is compact and disjoint from $f^{-1}(S)$, hence $\left\{z_{k}\right\}$ and $\left\{t_{k}\right\}$ cannot have a common limit point.

Proof of Theorem 2.1. Let $\lambda$ be the first ordinal with the cardinality of $Y$. We shall construct for each $\alpha<\lambda, m \in \mathbf{N}$ and $A \in \mathscr{A}$ an open $F_{\sigma}$ subset $F(\alpha, m, A)$ of $Y$, such that $\{F(\alpha, m, A)\}$ is a separating, $\sigma$-point finite family. Thus $Y$ is an $E-C$ by Rosenthal's characterization.

Construction of $F(\alpha, m, A)$. For a fixed $A \in \mathscr{A}$, the sets $S(A)$ and $U(A)$ satisfy the assumptions of Lemma 2.1, hence we can find for each $\alpha<\lambda$ and $m=1,2, \cdots$ an open subset $V(\alpha, m, A)$ of $U(A)$ and a compact subset $S(\alpha, m, A)$ of $S(A) \cap V(\alpha, m, A)$ such that

(a) $S(A)=\cup\{S(\alpha, m, A): \alpha<\lambda, m=1,2, \cdots\}$

(b) If $\alpha>\beta, S(\alpha, m, A) \cap V(\beta, n, A)=\varnothing$.

Fix now $\alpha, m, A$ such that $S(\alpha, m, A) \neq \varnothing$. Since $S(\alpha, m, A)$ is a compact subset of $V(\alpha, m, A)$, we can find by Lemma 2.3 a $k=$ $k(\alpha, m, A)$ such that whenever $z \in X$ is such that $\Gamma(z, k)=\Gamma(t, k)$ for some $t \in f^{-1}(S(\alpha, m, A))$, then $f(z) \in V(\alpha, m, A)$. Define $W=$ $W(\alpha, m, A)=\left\{z \in X: \exists t \in f^{-1}(S(\alpha, m, A)), \Gamma(z, k) \supseteq \Gamma(t, k)\right\} . \quad W$ is an open subset of $X$ which contains $f^{-1}(S(\alpha, m, A))$, thus $Z(\alpha, m, A)=$ $Y \backslash f(X \backslash W)$ is an open subset of $Y$ containing $S(\alpha, m, A)$. We now choose $F(\alpha, m, A)$ to be an open $F_{\sigma}$ set satisfying

$$
S(\alpha, m, A) \subset F(\alpha, m, A) \subset V(\alpha, m, A) \cap Z(\alpha, m, A) .
$$

Proof that $\{F(\alpha, m, A)\}$ separates points. Let $y \neq w$ be in $Y$. By Lemma 2.2 there is an $A \in \mathscr{A}$ such that $y \in S(A), w \notin U(A)$ (or visa versa). Since $S(A)=\bigcup\{S(\alpha, m, A): \alpha<\lambda, m=1,2, \cdots\}$ there are $\alpha$ and $m$ such that $y \in S(\alpha, m, A) \subset F(\alpha, m, A)$. Also $F(\alpha, m, A) \subset$ $V(\alpha, m, A) \subset U(A)$ hence $w \notin F(\alpha, m, A)$,

Proof that $\{F(\alpha, m, A)\}$ is $\sigma$-point finite. For each $\alpha, m, A$, let

$$
n(\alpha, m, A)=\max \{|A|, k(\alpha, m, A), l(A)\},
$$

where $l(A)=\max \left\{l: A \cap \Gamma_{l} \neq \varnothing\right\}$.

We shall show that for each fixed $m$ and $n$, the family $\{F(\alpha, m, A): n(\alpha, m, A) \leqq n\}$, is point-finite. Assume for contradiction that $y \in \bigcap_{j=1}^{\infty} F\left(\alpha_{l}, m, A_{l}\right)$ where $n\left(\alpha_{j}, m, A_{j}\right) \leqq n$ for all $j$. Since $k\left(\alpha_{j}, m, A_{j}\right) \leqq n$ we can assume, by passing to a subsequence, that $k\left(\alpha_{l}, m, A_{l}\right)=k$ is independent of $j$. Since $\left|A_{l}\right| \leqq n$ for all $j$, we can 
assume, by passing to a further subsequence and using Lemma 1.3 (a), that $\left\{A_{l}\right\}$ form a $\Delta$-system, i.e. there is a finite set $D \subset \Gamma$ such that $A_{i} \cap A_{j}=D$ for all $i \neq j$.

Fix any $z \in f^{-1}(y)$. Since $F\left(\alpha_{l}, m, A_{j}\right) \subset Z\left(\alpha_{l}, m, A_{j}\right)$, we get that $z \in f^{-1}\left(Z\left(\alpha_{l}, m, A_{l}\right)\right) \subset W\left(\alpha_{l}, m, A_{l}\right)$, and there is a $t_{j} \in f^{-1}\left(S\left(\alpha_{j}, m, A_{l}\right)\right)$ with $\Gamma(z, k) \supseteq \Gamma(t, k)$. Since $\Gamma(z, k)$ is a finite set, we can assume, by passing to a subsequence that $\Gamma\left(t_{i}, k\right)=\Gamma\left(t_{j}, k\right)$ for all $i$ and $j$, and by the definition of $k=k\left(\alpha_{j}, m, A_{j}\right)$, we get that $f\left(t_{i}\right) \in V\left(\alpha, m, A_{j}\right)$ for all $i$ and $j$.

We now distinguish two cases to get the desired contradiction:

Case 1. There are $i \neq j$ such that $A_{i}=A_{l}=D$. Then necessarily $\alpha_{\imath} \neq \alpha_{j}$ and assume $\alpha_{\imath}>\alpha_{j}$. But then $f\left(t_{\imath}\right) \in S\left(\alpha_{i}, m, D\right) \cap V\left(\alpha_{l}, m, D\right)$ contradicting Lemma 2.1 (b).

Case 2. All the $A_{j}$ 's are different from each other (and different from $D)$. Fix any $\gamma \in A_{1} \backslash D$. Since $f\left(t_{1}\right) \in S\left(\alpha_{1}, m, A_{1}\right) \subset S\left(A_{1}\right)$, there is a $\bar{t}_{1} \in f^{-1}\left(f\left(t_{1}\right)\right)$ such that $\Gamma\left(\bar{t}_{1}\right) \cap A_{1}=\{\gamma\}$. Since $\Gamma\left(\bar{t}_{1}, n\right)$ is a finite set and all the sets $\left\{A_{j} \backslash D\right\}$ are disjoint and contained in $\Gamma_{1} \cup \cdots \cup \Gamma_{n}$ (here we use the fact that $\left.l\left(A_{j}\right) \leqq n\right)$, we can find an $i$ such that $\left(A_{i} \backslash D\right) \cap$ $\Gamma\left(\bar{t}_{1}\right)=\varnothing$. But then also $A_{i} \cap \Gamma\left(\bar{t}_{1}\right)=\varnothing$, and we get that $f\left(\bar{t}_{1}\right) \notin U\left(A_{i}\right)$, contradicting the fact that $f\left(\bar{t}_{1}\right)=f\left(t_{1}\right) \in V\left(\alpha_{j}, m, A_{i}\right) \subset U\left(A_{i}\right)$.

We now pass to some Banach space consequences of Theorem 2.1. First recall some notation and definitions. If $X$ is a compact Hausdorff space, $C(X)$ denotes the Banach space of continuous functions on $X$, with the sup norm. For a Banach space $E$, we denote by $E^{*}$ its dual, and by $B\left(E^{*}\right)$ the unit ball of $E^{*}$ endowed with the $w^{*}$. topology. A Banach space is called $W C G$ (weakly compactly generated), if it is the closed linear span of a weakly compact subset.

Corollary 2.1. Let $E$ be a Banach space. Then $E$ is a subspace of a WCG space iff $B\left(E^{*}\right)$ is an $E-C$.

Proof. If $B\left(E^{*}\right)$ is an $E-C$, then $C\left(B\left(E^{*}\right)\right)$ is $W C G$ [1], and $E$ is canonically a subspace of $C\left(B\left(E^{*}\right)\right)$. Conversely, if $E$ is a subspace of a $W C G$ space $F$, then $B\left(F^{*}\right)$ is an $E-C$ [1], and the restriction map $R: B\left(F^{*}\right) \rightarrow B\left(E^{*}\right)$ is a $w^{*}$-continuous onto map. Thus by Theorem $2.1, B\left(E^{*}\right)$ is also an $E-C$.

H. P. Rosenthal [8], gave a example of a non- $W C G$ subspace of a $W C G$ space. The next corollary says that this cannot happen for $C(X)$ subspaces.

COROLlary 2.2. Let $F$ be WCG, and $E$ a subspace of $F$ which is isomorphic to a $C(X)$ space. Then $E$ is WCG. 
Proof. By Corollary $2.1, B\left(E^{*}\right)$ is an $E-C$, and a space $E$, isomorphic to $C(X)$, is $W C G$ iff $B\left(E^{*}\right)$ is an $E-C$ [1].

3. Uniform $\boldsymbol{E}-\boldsymbol{C}$. We start with the analogue of Theorem 2.1 for uniform $E-C$.

THEOREM 3.1. Let $X$ be a uniform $E-C$, and $f$ a continuous map from $X$ onto $Y$. Then $Y$ is also a uniform $E-C$.

Proof. We examine the proof of Theorem 2.1 and use the same notation By Lemma 1.2 we can assume that $X$ is a closed subset of $\mathscr{K}^{\mathrm{N}}$, i.e. $X \subset\{0,1\}^{\Gamma}, \Gamma=\bigcup_{n=1}^{\infty} \Gamma_{n}$ where the $\Gamma_{n}$ 's are disjoint and $\mid\{\gamma \in$ $\left.\Gamma_{n}: x(\gamma)=1\right\} \mid \leqq 1$ for all $x \in X$ and all $n$. In the last step of the proof we shall use Lemma 1.3 (b) instead of 1.3 (a), and we shall show that if $m$ and $n$ are fixed, no $y$ belongs to more then $n \cdot g\left(n, 2^{n}(n+1)\right)$ different $F\left(\alpha_{\jmath}, m, A_{\jmath}\right)$ 's.

If this were not the case, we could find $k$ and more than $g\left(n, 2^{n}(n+1)\right) j$ 's for which $k\left(\alpha_{l}, m, A_{l}\right)=k$ is independent of $j$. By Lemma $1.3(\mathrm{~b})$ we can find a set $D$ and more than $2^{n}(n+1) A_{\jmath}$ 's for which $A_{\imath} \cap A_{,}=D$ if $i \neq j$.

Since $\Gamma(z, k)$ has cardinality at most $n$, there are $n+1 j$ 's for which $\Gamma\left(t_{l}, k\right)$ is independent of $j$, i.e. $f\left(t_{l}\right) \in V\left(\alpha_{l}, m, A_{l}\right)$ for all $i, j$. Case 1 is the same as in Theorem 2.1. In Case 2, since $\Gamma\left(t_{1}, n\right)$ has at most $n$ elements, and we have $n+1$ disjoint sets $\left\{A_{j} \backslash D\right\}$, there is an $i \neq 1$ with $\left(A_{i} \backslash D\right) \cap \Gamma\left(\bar{t}_{1}\right)=\varnothing$, which leads to the contradiction.

Our next result characterizes uniform $E-C$ in terms of their space of continuous functions. The equivalence of (a) and (d) is analogous to the equivalence of (a) and (c) in Theorem 2 in [1]. The equivalence of (a) and (c) should be compared with the theorem in [3] that a Banach space $E$ is $W C G$ iff there is an operator with dense range from a reflexive space into $E$.

THEOREM 3.2. Let $X$ be a compact Hausdorff space. The following are equivalent:

(a) $X$ is a uniform $E-C$.

(b) There is a dense range operator from a Hilbert space into $C(X)$.

(c) There is a dense range operator from some super-reflexive space into $C(X)$.

(d) $B\left(C(X)^{*}\right)$ is a uniform $E-C$.

Proof. Obviously (b) $\Rightarrow$ (c) and (d) $\Rightarrow$ (a). To see that (c) $\Rightarrow(d)$, let $T: F \rightarrow C(X)$ be a dense range operator from the super-reflexive space $F$. Then $T^{*}$ is one-one and $w^{*}$ continuous, hence a 
homeomorphism from $B\left(C(X)^{*}\right)$ into the super-reflexive space $F^{*}$. Thus (d) follows from the theorem in [2].

We now pass to the only nonobvious implication (a) $\Rightarrow(b)$. By the same argument as (2) $\Rightarrow(1)$ in the theorem of [2], we can assume that $X$ is a subset of $l_{2}(\Gamma)$ satisfying:

(1) $0 \notin X$

(2) $\sum_{\gamma \in \Gamma}|x(\gamma)| \leqq 1$ for all $x \in X$.

Let $\left\{e_{\gamma}\right\}$ be the standard basis for $l_{2}(\Gamma)$, and $\mathscr{A}_{n}=\Gamma \times \cdots \times \Gamma$ ( $n$ times). Let $\mathscr{A}=\cup_{n=0}^{\infty} \mathscr{A}_{n}\left(\mathscr{A}_{0}=\{\varnothing\}\right)$, and $H=\left(\sum_{\mathscr{A}} \oplus l_{2}(\Gamma)\right)_{2}$ and define $T: H \rightarrow C(X)$ as follows: If $h=\left(h_{A}\right)_{A \in \mathscr{A}} \in H$ and $x \in X$ define

$$
(T h)(x)=\sum_{n} 2^{-n}\left(\sum_{A \in \mathscr{A}_{n}}\left(\prod_{A=\left(\alpha_{1},,, \alpha_{n}\right)} e_{\alpha_{j}}(x)\left\langle h_{A}, x\right\rangle\right)\right) .
$$

$T$ is a bounded linear operator, because for each $x \in X$, if $\|h\| \leqq 1$ we also have $\left\langle h_{A}, x\right\rangle \leqq\left\|h_{A}\right\| \leqq\|h\| \leqq 1$ for all $A$, and hence by (2) we get

$$
\begin{aligned}
\left|\sum_{A \in \mathscr{A}_{n}} \prod_{A=\left(\alpha_{1},, \alpha_{n}\right)} e_{\alpha_{j}}(x)\left\langle h_{A}, x\right\rangle\right| & \leqq \sum_{A \in \mathscr{A}_{n}}\left|\prod_{A=\left(\alpha_{1}, \cdots, \alpha_{n}\right)} e_{\alpha_{j}}(x)\right| \\
& =\left(\sum_{\gamma \in \Gamma}\left|e_{\gamma}(x)\right|\right)^{n} \leqq 1
\end{aligned}
$$

But $T H$ contains all the polynomials in the coordinate functions $e_{\gamma}(x)$. Since these polynomials separate points in $X$ and do not have a common zero (by (1)), $T H$ is dense in $C(X)$ by the Stone-Weierstrass theorem.

Analogous to Corollaries 2.1 and 2.2 we get:

Corollary 3.1. A Banach space $E$ is a subspace of a $C(X)$ space, where $X$ is a uniform $E-C$, iff $B\left(E^{*}\right)$ is a uniform $E-C$. Moreover, if $E$ is itself a $C(Y)$ space, this happens iff $Y$ is a uniform $E-C$.

4. Some topological properties of $\boldsymbol{E}-\boldsymbol{C}$. The first deep result on the topological structure of weakly compact sets in Banach spaces, and the origin of the name "Eberlein compacts", is the following famous theorem of Eberlein and Smulian (see [4]).

THEOREM 4.1. Let $X$ be an $E-C$ and $Y$ a subset of $X$. If $x_{0} \in \bar{Y}$, then there is a sequence in $Y$ converging to $x_{0}$.

It should be remarked that this theorem is trivial for weakly compact subsets of $c_{0}(\Gamma)$. However, the Amir-Lindenstrauss theorem which says 
that every $E-C$ is homeomorphic to a weakly compact subset of $c_{0}(\Gamma)$, is much deeper than the Eberlein-Smulian theorem.

The usual cardinal functions of an $E-C$ are easy to compute. In fact they are the smallest cardinality of a set $\Gamma$ such that $X$ embeds in $c_{0}(\Gamma)$. We shall prove one such result, which implies many others.

Definition. Let $X$ be a topological space. The cellularity of $X$, is the supremum of all cardinals $\lambda$ such that $X$ contains $\lambda$ pairwise disjoint open sets. If the cellularity of $X$ is $\boldsymbol{N}_{0}$, we say that $X$ is CCC (countable chain condition).

THEOREM 4.2. Let $X$ be an $E-C$ and $\lambda$ the smallest cardinality of a set $\Gamma$, such that $X$ embeds in $c_{0}(\Gamma)$. Then the cellularity of $X$ is $\lambda$.

Proof. Assume $X \subset c_{0}(\Gamma)$ with $\mid \Gamma !=\lambda$, and fix any cardinal $\mu<\lambda$. Since $\lambda$ is minimal, there is an $\epsilon>0$ such that $\mathscr{A}=\{A \subset \Gamma: \exists x \in X$, $x(\gamma)>\epsilon \forall \gamma \in A\}$ has cardinality at least $\mu$. A does not contain an infinite increasing chain $A_{n}$, because any limit point of the appropriate $x_{n}$ 's, would be at least $\epsilon$ on the infinite set $\cup A_{n}$. Thus every $A \in \mathscr{A}$ is contained in a maximal one. Let $\mathscr{B}$ be the set of maximal elements in $\mathscr{A}$. By the above $|\mathscr{B}| \geqq \mu$ and for each $B \in \mathscr{B}$ the set $U_{B}=$ $\{x \in X: \forall \gamma \in B,|x(\gamma)|>\epsilon\}$ is open and nonempty. The sets $\left\{U_{B}\right\}_{B \in \mathscr{B}}$ are pairwise disjoint because if $x \in U_{B_{1}} \cap U_{B_{2}}$ then $|x(\gamma)|>\epsilon$ for $\gamma \in B_{1} \cup B_{2}$ contradicting their maximality.

REMARKS. (a) It is clear from our proof that if $\lambda$ is not the limit of a sequence of smaller cardinals, then in fact one can find $\lambda$ pairwise disjoint open sets in $X$. The same is true, however, also if $\lambda$ is a limit of a sequence of smaller cardinals by a general theorem in point-set topology (see [6] p. 37).

(b) The special case of the theorem, saying that if $X$ is not separable it is not CCC, was proved by Rosenthal [9] using different methods.

Our next result is a stronger form of Theorem 5 in [1]. Our proof is entirely different.

THEOREM 4.3. Every $E-C$ contains a dense $G_{\delta}$ metrizable set of $G_{\delta}$ points.

Proof. We start with the simple remark that a strong $E-C$ does not contain a perfect subset. Indeed if $X \subset\{0,1\}^{\Gamma}$ is a strong $E-C$ and $Z \subset X$, let $\mathscr{A}=\left\{A \subset \Gamma: \chi_{A} \in Z\right\}$, where $\chi_{A}$ is the indicator function of $A$. A does not contain an infinite increasing chain, hence there is a 
maximal element $B$ in $\mathscr{A}$. But then $\chi_{B}$ is an isolated point in $Z$. (This fact was also proved in [8], page 109.)

We now prove the theorem for the special case of an $E-C, X$, which is a closed subset of $\prod_{k=1}^{\infty} X_{k}$ where each $X_{k}$ is a strong $E-C$. Denote by $R_{n}: X \rightarrow \prod_{k=1}^{n} X_{k}$ the natural projection and let $D_{n}=$ $\left\{x \in X: R_{n}^{-1}\left(R_{n}(x)\right)\right.$ is open in $\left.X\right\}$. Clearly $D=\bigcap_{m=1}^{\infty} \bigcup_{n=m}^{\infty} D_{n}$ is a $G_{\delta}$ set in $X$ consisting of $G_{\delta}$ points.

Define a metric $\rho$ on $D$ by $\rho(x, y)=\left[\min \left\{n: R_{n} x \neq R_{n} y\right\}\right]^{-1}$. If $\rho\left(x_{n}, x\right) \rightarrow 0$ clearly $x_{n} \rightarrow x$. However if $x \in D_{m}$ and $x_{n} \rightarrow x$ then necessarily $R_{m}(x)=R_{m}\left(x_{n}\right)$ for large enough $n$ and hence $\rho\left(x_{n}, x\right) \leqq$ $1 / m$. Since $x \in D$ iff $x \in D_{m}$ for infinitely many $m$ 's, we get that if $x_{n} \rightarrow x$ and $x \in D$ then $\rho\left(x_{n}, x\right) \rightarrow 0$, i.e. $\rho$ induces the original topology on $D$.

To see that $D$ is dense, it is enough, by Baire's theorem to show that $\cup_{n=m}^{\infty} D_{n}$ is dense for every $m$. Thus let $U$ be a basic open set depending on the first $N$ coordinates, and assume $N \geqq m$. Let $x \in U$ be such that $R_{N} x$ is isolated in $R_{n}(U)$ (such an $x$ exists by the remark in the beginning of the proof, since $\Pi_{k=1}^{N} X_{k}$ is a strong $\left.E-C\right)$. We then have $x \in D_{N}$, i.e. $\left(\bigcup_{n=m}^{\infty} D_{n}\right) \cap U \neq \varnothing$.

To prove the general case, let $Y$ be any $E-C$. By Lemma 1.1 we can find a closed subset $X$ of a countable product of strong $E-C$ 's $\left\{X_{k}\right\}$, and an onto map $f: X \rightarrow Y$. By Zorn's Lemma we can assume that $f$ is irreducible (i.e. $f\left(X_{1}\right) \neq Y$ for all closed proper subsets $X_{1}$ of $X$ ). Let $D_{n}$ and $D$ in $X$ be as in the first part of the proof.

For each $x \in D_{n}$, let $U(x, n)=f^{-1}\left[Y \backslash f\left(X \backslash R_{n}^{-1} R_{n}(x)\right)\right] . \quad U(x, n)$ is an open subset of $X$ which is non-empty by the irreducibility of $f$, also $f^{-1}(f(U(x, n)))=U(x, n)$.

Let $E_{n}=\cup\left\{U(x, n): x \in D_{n}\right\} . \quad E_{n}$ is an open subset of $D_{n}$, and again by the irreducibility of $f, \cup_{n=m}^{\infty} E_{n}$ is dense in $X$ for all $m$. Thus $E=\bigcap_{m=1}^{\infty} \bigcup_{n=m}^{\infty} E_{n}$ is a dense $G_{\delta}$ subset of $D$ such that $f^{-1}(f(E))=$ $E$. Also if $e \in E$ there is an increasing sequence $\left\{n_{j}\right\}$ such that $e \in$ $U\left(x_{j}, n_{j}\right)$, and thus also $f^{-1}(f(e)) \subset U\left(x_{j}, n_{j}\right)$. But clearly $\bigcap_{j=1}^{\infty} U\left(x_{j}, n_{j}\right)$ cannot contain more than one point, hence $f^{-1}(f(e))=e$. Using the fact that if $K$ is $G_{\delta}$ in $X$ and $f^{-1}(f(K))=K$ then $f(K)$ is $G_{\delta}$ in $Y$, we thus get that $f(E)$ is a dense $G_{\delta}$ subset of $Y$ consisting of $G_{\delta}$ points. It is also easy to check that $f$ is a homeomorphism on $E$, and thus $f(E)$ is metrizable.

5. Examples. By Rosenthal's characterization, a compact Hausdorff space is an $E-C$ if it has a $\sigma$-point-finite separating family of open $F_{\sigma}$ 's.

It was observed by Michael (see [8]), that one cannot replace the condition of separation by strong separation. (A family $\mathscr{F}$ is strongly separating, if given $x \neq y$ in $X$, there is an $F \in \mathscr{F}$ with $x \in F, y \notin F)$. In 
fact, a compact Hausdorff space admitting a strongly separating, point countable family of open $F_{\sigma}$ 's is metrizable. (A family $\mathscr{F}$ is point countable if every $x \in X$ belongs to at most countably many members of $\mathscr{F}$. Clearly every $\sigma$-point-finite family is point countable).

A very simple example was given in [11] of a compact Hausdorff space which is not an $E-C$ but has a $\sigma$-point-finite, separating family of open (but not $F_{\sigma}$ ) subsets. We just mention here without giving the construction that there exists a compact Hausdorff space $X$ which is not an $E-C$ but has the following properties

(1) $X$ has a $\sigma$-point finite separating family of open sets.

(2) If $Y \subset X$ and $x_{0} \in \bar{Y}$, there is a sequence in $Y$ converging to $x_{0}$.

(3) A closed subset of $X$ is metrizable iff it is separable iff it is CCC.

(4) $X$ contains a metrizable dense open subset of isolated points. (Properties (2)-(4) should be compared with the properties of $E-C$ proved in $\S 4)$.

One also cannot replace the assumption of $\sigma$-point-finite by the weaker assumption that the family is point-countable. Recall that a Souslin interval is a linearly ordered topological space (with the order topology), which is compact, connected, CCC and non-separable. The existence of a Souslin interval is known to be independent of the usual axioms of set theory (see e.g. [10]), and it is quite plausible that under different set theoretic assumption one could replace the $\sigma$-point finite condition by point-countability.

ExAmPle 5.1. Assume that Souslin interval exists. Then there is a compact Hausdorff space which is not an $E-C$, but has a pointcountable separating family of open $F_{\sigma}$ 's.

The construction. Let $L$ be a Souslin interval. Identify every maximal closed separable subinterval to a single point. The resulting space, $L^{*}$, is CCC, compact, non-separable, connected, linearly ordered, and has no non-trivial separable subinterval.

By transfinite induction, choose for each countable ordinal $\alpha$ a family $\mathscr{L}_{\alpha}$ of disjoint open subintervals of $L^{*}$ satisfying

(a) For each $\alpha, \cup \mathscr{L}_{\alpha}$ is dense in $L^{*}$.

(b) If $\beta<\alpha$ and $I \in \mathscr{L}_{\alpha}$, there is a $J \in \mathscr{L}_{\beta}$ with $I \subset J$.

(c) If $\alpha=\beta+1$ and $J \in \mathscr{L}_{\beta}$, then $\left\{I \in \mathscr{L}_{\alpha}: I \subset J\right\}$ is order isomorphic (in the natural order) to the rationals (in the reals).

(d) If $\alpha$ is a limit ordinal and $I \in \mathscr{L}_{\alpha}$, then

$$
\bar{I}=\bigcap_{\beta<\alpha}\left\{J: J \in \mathscr{L}_{\beta}, J \supset I\right\} .
$$

Now if $J \in \mathscr{L}_{\beta}$ then $R_{J}=J \backslash \cup\left\{I \in \mathscr{L}_{\beta+1}: I \subset J\right\}$ is order isomorphic 
to the irrationals (in the reals), and thus there is a countable family $\mathscr{F}_{J}$ of open subintervals of $J$ which separates the points in $R_{J}$.

Notice that the family $\mathscr{F}_{F}=\bigcup_{J} \mathscr{F}_{J}$ is point-countable, because every nested family of intervals in $L^{*}$ is at most countable, by the fact that $L^{*}$ is CCC. Hence given any $t \in L^{*}$, there are at most countably many $\beta$ 's and $J_{\beta} \in \mathscr{L}_{\beta}$ with $t \in J_{\beta}$.

Also $\mathscr{F}$ separates every pair of points $x \neq y$ in $L^{*}$ provided they are not the end points of the same interval $I$ in some $\mathscr{L}_{\alpha}$, where $\alpha$ is a limit ordinal.

The Souslin Cactus $X$ is the quotient space obtained from $L^{*}$ by identifying each pair of end points of every interval $I \in \mathscr{L}_{\alpha}$ for all limit ordinals $\alpha$ to single points. $X$ is a compact Hausdorff space and $\cup_{J} \mathscr{F}_{J}$ is a point-countable, separating family of open $F_{\sigma}$ 's in $X$. However $X$ is nonmetrizable and is CCC, hence it cannot be an $E-C$ (by Theorem 4.2).

Our last example gives a negative answer to a question of $\mathrm{H}$. P. Rosenthal [8].

EXAMPLE 5.2. There exists a non-metrizable $E-C$, in which every closed metrizable subset is $G_{\delta}$.

The construction. Let $S=\{0,1,1 / 2,1 / 3, \cdots\}$ with the usual topology, and denote by $\omega_{1}$ the first uncountable ordinal. We shall construct inductively for each $\alpha<\omega_{1}$, a point $x_{\alpha} \in S^{\omega_{1}}$ satisfying

(1) $\sum_{\beta<\omega_{1}} x_{\alpha}(\beta)<1$

(2) $\Gamma\left(x_{\alpha}\right)=\{\gamma: \gamma \leqq \alpha\} \quad$ (where for $\quad x \in S^{\omega_{1}}, \Gamma(x)=\{\gamma \in$ $\left.\left.\omega_{1}: x(\gamma) \neq 0\right\}\right)$.

(3) If $x_{\alpha}(\delta)=x_{\beta}(\delta)$ for some $\delta \leqq \alpha \leqq \beta$, then $x_{\alpha}(\gamma)=x_{\beta}(\gamma)$ for all $\gamma \leqq \delta$.

Once these $x_{\alpha}$ 's are constructed, let $X$ be the closure of $\left\{x_{\alpha}\right\}$ in $S^{\omega_{1}}$ (with the product topology). By (1) $X$ is an $E-C$, and it has the following property: If $x \in X$ and $x(\gamma)=0$, then $\Gamma(x) \subset[1, \gamma)=\{\alpha<$ $\left.\omega_{1}: \alpha<\gamma\right\}$. Indeed, if $x(\delta) \neq 0$ for some $\delta>\gamma$, then $x(\delta)=1 / n$ for some $n$, but then if $x_{\alpha_{j}} \rightarrow x$, then also $x_{\alpha_{j}}(\delta)=1 / n$ for $j$ large enough. Hence by (3) $x_{\alpha_{j}}(\gamma)=x_{\alpha_{i}}(\gamma)$ for all large enough $i$ and $j$. But then $x_{\alpha_{1}}(\gamma)=\lim _{i} x_{\alpha_{t}}(\gamma)=x(\gamma)=0$, and $x_{\alpha_{1}}(\delta)=1 / n \neq 0$ contradicting (2).

In particular, for each $\gamma<\omega_{1}$ the set

$$
X_{\gamma}=\{x \in X: \Gamma(x) \subset[1, \gamma)\}=\{x \in X: x(\gamma)=0\}=\bigcap_{n=1}^{\infty}\left\{x: x(\gamma)<\frac{1}{n}\right\}
$$

is a $G_{\delta}$ set which is clearly metrizable. But every closed metrizable subset of $X$ is separable and is thus contained in some $X_{r}$, hence it is also $G_{\delta}$. 
In order to construct the $x_{\alpha}$ 's, we add another assumption:

(4) For every limit ordinal $\lambda<\omega_{1}$ and every $\beta<\lambda$, there is a sequence $\alpha_{n}$ increasing to $\lambda, \alpha_{n}>\beta$ such that

(a) $x_{\alpha_{n}}(\gamma)=x_{\beta}(\gamma)$ for all $\gamma \leqq \beta$

(b) $\sum_{\gamma<\omega_{1}} x_{\alpha_{n}}(\gamma) \leqq \frac{1}{2^{n}}+\sum_{\gamma<\omega_{1}} x_{\beta}(\gamma)$

We construct the $x_{\alpha}$ 's inductively in "blocks": If $\lambda$ is a limit ordinal, and $\left\{x_{\alpha}\right\}_{\alpha<\lambda}$ are already defined satisfying (1)-(4), we construct $x_{\alpha}$ 's for $\lambda \leqq \alpha<\lambda+\omega$. The details are left to the reader.

6. Open problems. The first four problems deal with universal $E-C$.

Problem 1. Given a cardinal $\lambda$, does there exist an $E-C, X$, of weight $\lambda$, such that every $E-C$ of weight $\lambda$ is a continuous image of $X$ ?

Problem 2. Given a cardinal $\lambda$, does there exist a $W C G$ Banach space $E$ of density character $\lambda$ such that every $W C G$ space of density character $\lambda$ embeds in $E$ ?

It is clear that a positive answer to 1 will also give a positive answer to 2 .

Problem 3. Given a cardinal $\lambda$, does there exist a uniform $E-C$, $X$, of weight $\lambda$, such that every uniform $E-C$ of weight $\lambda$ is a continuous image of $X$ ?

By Lemma 1.2 , every uniform $E-C$ is a continuous image of a closed subset of $\mathscr{K}^{\mathrm{N}}$.

Problem 4. Is $\mathscr{K}^{\mathrm{N}}$ universal for uniform $E-C$ ?

Even if the answer to problem 4 is negative, its solution will involve better understanding of $\mathscr{K}^{\mathbf{N}}$ whose topological structure deserves a detailed study.

Problem 5. Does there exist a nonmetrizable homogeneous $E-C ?$

Recall that a topological space $X$ is called homogeneous if for every $x, y \in X$ there is a homeomorphism $f$ of $X$ onto itself which carries $x$ to $y$.

We observed in the beginning of the proof of Theorem 4.3 that a strong $E-C$ is scattered.

Problem 6. Is every scattered $E-C$ a strong $E-C$ ? 
Our last problem is related to Corollary 2.1. It is not clear however whether it has any connection with the material of this paper.

Answering a problem of Corson (see [7] problem 2), M. Talagrand [12] has recently shown that a $W C G$ Banach space is Lindelöf in its weak topology. The property of being Lindelöf in the weak topology is inherited by closed subspaces, however, as Rosenthal has shown [8], the property of being WCG is not. We thus reformulate the other implication of Corson's problem.

Problem 7. Is a Banach space $E$ a subspace of a $W C G$ space if it is Lindelöf in its weak topology?

The reader is referred to [7] Problems 6 and $6^{\prime}$ for related questions.

Added in proof. Problems 6 and 7 are now solved. K. Alster (Some remarks on Eberlein compacts, to appear) showed that the answer to problem 6 is positive. R. Pol (A function space $C(X)$ which is weakly Lindelof but not weakly compactly generated, to appear), and M. Talagrand (Espaces de Banach Faiblement -analytiques, C. R. Paris, 284, 1977, 745-748) have independently given counter-examples to problem 7.

\section{REFERENCES}

1. D. Amir and J. Lindenstrauss, The structure of weakly compact sets in Banach spaces, Ann. of Math., 88 (1968), 34-46.

2. Y. Benyamini and T. Starbird, Embedding weakly compact sets into Hilbert space, Israel J. Math., 23 (1976), 137-141.

3. W. J. Davis, T. Figiel, W. B. Johnson, and A. Pelczynski, Factoring weakly compact operators, J. Functional Analysis, 17 (1974), 311-327.

4. N. Dunford and J. Schwartz, Linear operators, Vol. I, Interscience, N.Y., 1958.

5. P. Erdös and R. Rado, Intersection theorems for systems of sets, J. London Math. Soc., 35 (1960), 85-90.

6. I. Juhasz, Cardinal functions in topology, Math. Centrum Tract. No. 34, Amsterdam, 1971.

7. J. Lindenstrauss, Weakly compact sets, their topological properties and the Banach spaces they generate, Annals of Math. Studies 69, Princeton Univ. Press, (1972), 235-273.

8. H. P. Rosenthal, The heredity problem for weakly compactly generated Banach spaces, Compositio Math., 28 (1974), 83-111.

9. _ On injective Banach spaces and the spaces $L^{\infty}(\mu)$ for finite measures $\mu$, Acta Math., 124 (1970), 205-248.

10. M. E. Rudin, Lectures in set theoretic topology, CBMS regional conference series in mathematics, No. 23 (1974).

11. P. Simon, On continuous images of Eberlein compacts, Comment. Math. Univ. Carolinae, 17 (1976), 179-194. 
12. M. Talagrand, Sur une conjecture de H. H. Corson, Bull. Sci. Math., 99 (1975), 211-212.

Received September 15, 1976. The first author was partially supported by NSF grant MPS-74-2449 and the second author was partially supported by NSF grant MPS-73-08825.

Yale University, New Haven, CT 06520

AND

UNIVERSITY OF WISCONSIN-MADISON, WI 53706

Current addresses: Y. Benyamini, Technion, Institute of Technology, Haifa, Israel

M. E. Rudin, University OF Wisconsin, Madison, WI 53706.

M. Wage, Yale University, New Haven, CT. 06520. 


\section{PACIFIC JOURNAL OF MATHEMATICS \\ EDITORS}

RICHARD ARENS (Managing Editor)

University of California

Los Angeles, CA 90024

\section{R. A. Beaumont \\ University of Washington \\ Seattle, WA 98105}

C. C. MOORE

University of California

Berkeley, CA 94720

\section{J. DUGUNDJI}

Department of Mathematics

University of Southern California

Los Angeles, CA 90007

R. FINN AND J. MILGRAM

Stanford University

Stanford, CA 94305

\section{ASSOCIATE EDITORS}

\section{E. F. BECKENBACH}

B. H. NeUmanN

F. WOLF

K. YoSHIDA

\section{SUPPORTING INSTITUTIONS}

\author{
UNIVERSITY OF BRITISH COLUMBIA \\ CALIFORNIA INSTITUTE OF TECHNOLOGY \\ UNIVERSITY OF CALIFORNIA \\ MONTANA STATE UNIVERSITY \\ UNIVERSITY OF NEVADA \\ NEW MEXICO STATE UNIVERSITY \\ OREGON STATE UNIVERSITY \\ UNIVERSITY OF OREGON \\ OSAKA UNIVERSITY
}

\author{
UNIVERSITY OF SOUTHERN CALIFORNIA \\ STANFORD UNIVERSITY \\ UNIVERSITY OF HAWAII \\ UNIVERSITY OF TOKYO \\ UNIVERSITY OF UTAH \\ WASHINGTON STATE UNIVERSITY \\ UNIVERSITY OF WASHINGTON \\ AMERICAN MATHEMATICAL SOCIETY
}

The Supporting Institutions listed above contribute to the cost of publication of this Journal, but they are not owners or publishers and have no responsibility for its contents or policies.

Mathematical papers intended for publication in the Pacific Journal of Mathematics should be in typed form or offset-reproduced (not dittoed), double spaced with large margins. Underline Greek letters in red, German in green, and script in blue. The first paragraph or two must be capable of being used separately as a synopsis of the entire paper. Items of the bibliography should not be cited there unless absolutely necessary, in which case they must be identified by author and Journal, rather than by item number. Manuscripts, in duplicate, may be sent to any one of the four editors. Please classify according to the scheme of Math. Reviews, Index to Vol. 39. All other communications should be addressed to the managing editor, or Elaine Barth, University of California, Los Angeles, California, 90024.

100 reprints are provided free for each article, only if page charges have been substantially paid. Additional copies may be obtained at cost in multiples of 50 .

The Pacific Journal of Mathematics is issued monthly as of January 1966. Regular subscription rate: $\$ 72.00$ a year (6 Vols., 12 issues). Special rate: $\$ 36.00$ a year to individual members of supporting institutions.

Subscriptions, orders for back numbers, and changes of address should be sent to Pacific Journal of Mathematics, 103 Highland Boulevard, Berkeley, California, 94708.

PUBLISHED BY PACIFIC JOURNAL OF MATHEMATICS, A NON-PROFIT CORPORATION

Printed at Jerusalem Academic Press, POB 2390, Jerusalem, Israel.

Copyright (C) 1977 Pacific Journal of Mathematics All Rights Reserved 


\section{Pacific Journal of Mathematics \\ Vol. 70, No. $2 \quad$ October, 1977}

B. Arazi, A generalization of the Chinese remainder theorem ........... 289

Thomas E. Armstrong, Polyhedrality of infinite dimensional cubes .... . . . 297

Yoav Benyamini, Mary Ellen Rudin and Michael L. Wage, Continuous

images of weakly compact subsets of Banach spaces ............ 309

John Thomas Burns, Curvature functions on Lorentz 2-manifolds ......... 325

Dennis F. De Riggi and Nelson Groh Markley, Shear distality and equicontinuity .................................. 337

Claes Fernström, Rational approximation and the growth of analytic

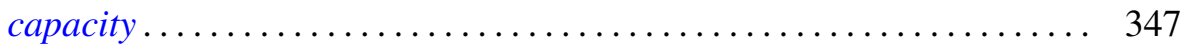

Pál Fischer, On some new generalizations of Shannon's inequality....... 351

Che-Kao Fong, Quasi-affine transforms of subnormal operators ......... 361

Stanley P. Gudder and W. Scruggs, Unbounded representations of

*-algebras........................................ 369

Chen F. King, A note on Drazin inverses .................... 383

Ronald Fred Levy, Countable spaces without points of first countability . . . 391

Eva Lowen-Colebunders, Completeness properties for convergence

spaces ......................................... 401

Calvin Cooper Moore, Square integrable primary representations ....... 413

Stanisław G. Mrówka and Jung-Hsien Tsai, On preservation of

E-compactness ................................ 429

Yoshiomi Nakagami, Essential spectrum $\Gamma(\beta)$ of a dual action on a von

Neumann algebra ................................ 437

L. Alayne Parson, Normal congruence subgroups of the Hecke groups

$G\left(2^{(1 / 2)}\right)$ and $G\left(3^{(1 / 2)}\right)$...

Louis Jackson Ratliff, Jr., On the prime divisors of zero in form rings . . . . 489

Caroline Series, Ergodic actions of product groups .................. 519

Robert O. Stanton, Infinite decomposition bases..................... 549

David A. Stegenga, Sums of invariant subspaces .................. 567 